\title{
Equivalence problem and integrability of the Riccati equations
}

\author{
Tomasz Czyżycki and Jiří Hrivnák
}

\begin{abstract}
We consider the class of general real Riccati equations and find its Lie group of equivalence transformations. Using the Lie algebra of this Lie group and its invariants we formulate criteria of equivalence of the Riccati equations. These criteria determine some cases of the general Riccati equations, which are integrable in quadratures.
\end{abstract}

Mathematics Subject Classification (2000). 34C41, 34C14.

Keywords. General Riccati equation, Equivalence transformations, Invariants.

\section{Introduction}

The Riccati equation is of a great importance in the theory of differential equations and its applications. It is known that many problems in mathematics and physics can be reduced to Riccati equation e.g. the second order linear ordinary differential equation $y^{\prime \prime}+q_{1}(x) y^{\prime}+q_{2}(x) y=0$, the Schrödinger equation and others [1]. The main difficulty by solving these problems lies in the fact, that not every Riccati equation has the general solution in an explicit form. From the symmetry point of view difficulties in solving Riccati equations are created by the absence of common explicit symmetries of these equations.

The notion of equivalence transformations is the generalization of symmetry and it is defined for a class of differential equations. It allows to investigate some properties of this class in a local context and find the local change of variables that transforms one equation to another. The equivalence transformations have already been used by Lie [2] for group classification of the second order ordinary differential equations. The invariants of equivalence transformations and infinitesimal technique of finding these invariants has been introduced by Ovsiannikov [3,4]. It is known that invariants and differential invariants of Lie group of symmetry of differential equation and Lie group of equivalence transformations of a class of differential equations are very 
important in investigations of their properties such as reduction and invariant form [5-9], as well as isomorphisms of the studied Lie algebras [10].

In this paper we find the Lie group of equivalence transformations of the class of the general real Riccati equations and study its properties. Using this group we formulate criteria of equivalence and integrability of the Riccati equations. These criteria are based on locally defined invariants and differential invariants of equivalence transformations, so they also work locally. This new approach allows us to avoid difficult calculations and to compare only few simple expressions to establish whether two equations are equivalent or not. If the equivalence conditions hold it is possible in some cases to construct exact transformations which lead one Riccati equation to another. In particular it is important to formulate conditions for their equivalence, by the action of certain fixed subgroups of the total equivalence group, to Bernoulli equation. This allow us to integrate them by quadratures provided that equivalence transformations can be found in explicit way.

In Sect. 2, we define the equivalence transformations of a class of differential equations and formulate their properties. Moreover we formulate the Tresse theorem for function $y$ depending on one independent variable.

In Sect. 3, using the infinitesimal technique of finding the Lie algebra of a Lie group, we find the Lie group of equivalence transformations of the class of the general Riccati equations.

In Sect. 4, following the procedure based on the Tresse theorem, we construct invariants, differential invariants and invariant differentiation operators for each subgroup of the previously found Lie group of equivalence transformations.

In Sect. 5, using found invariants, we formulate criteria of equivalence of Riccati equations.

In Sect. 6, we describe some integrable cases and formulate criteria of integrability of the general Riccati equation. Concluding remarks are presented in Sect. 7.

\section{Equivalence transformations and their properties}

In this section, we define the basic notions from group-theoretical analysis of differential equations following Ovsiannikov [11], Olver [12,13] and Ibragimov $[14,15]$. Among these we define equivalence transformations of a class of differential equations.

Let $y$ denotes a real function $y(x)$ and $y^{\prime}, y^{\prime \prime}, \ldots, y^{(k)}$ denote the derivatives of function $y(x)$ with respect to $x, D_{x}$ denotes the operator of the total derivative with respect to $x, D_{x}=\frac{\partial}{\partial x}+y^{\prime} \frac{\partial}{\partial y}+y^{\prime \prime} \frac{\partial}{\partial y^{\prime}}+\cdots$ and $D_{x}^{k}$ denotes the total derivative $k$ times with respect to $x$.

Consider the action of a Lie group of transformations $G$ in the space of variables $(x, y)$, where $y$ is a dependent variable and corresponding infinitesimal generator $X=\xi(x, y) \partial_{x}+\eta(x, y) \partial_{y}$ in the Lie algebra of vector fields of a Lie group $G$. 
We define the extension of the $m$ th order $\underset{m}{X}$, of the operator $X$ to the space $\left(x, y, y^{\prime}, \ldots, y^{(m)}\right)$ by:

$$
\underset{m}{X}=X+\sum_{k=1}^{m} \zeta^{k} \partial_{y^{(k)}},
$$

where coefficients $\zeta^{k}$ are defined recursively:

$$
\zeta^{k}=D_{x}\left(\zeta^{k-1}\right)-y^{(k)} D_{x}(\xi) .
$$

$k=1,2, \ldots$ and we mean $\zeta^{0}=\eta$.

Definition 2.1. Let $G$ be a Lie group of transformations of the space $(x, y)$. We denote by $\underset{m}{G}$ the extension of the group $G$ to the space $\left(x, y, y^{\prime}, \ldots, y^{(m)}\right)$.

a) A function $F(x, y)$ is called an invariant of $G$ iff :

$$
\forall_{\varphi \in G} F(\varphi(x, y))=F(x, y) \text {. }
$$

b) A function $F\left(x, y, y^{\prime}, \ldots, y^{(m)}\right)$ is called a differential invariant (of the $m$ th order) of the group $G$ iff :

$$
\forall_{\varphi} \in G \in \underset{m}{ } F\left(\underset{m}{\varphi}\left(x, y, y^{\prime}, \ldots, y^{(m)}\right)\right)=F\left(x, y, y^{\prime}, \ldots, y^{(m)}\right) .
$$

c) The general (or universal) differential invariant of the $m$ th order is the set of all differential invariants from the order zero to the order $m$ inclusive,

d) A maximal set of functionally independent invariants of the order $r \leq m$ of a Lie group $G$ is called a functional basis of the $m$ th order differential invariants of $G$,

e) $Q$ is called an operator of the invariant differentiation, if for any differential invariant $F$ of the group $G$ the expression $Q F$ is also the differential invariant of the group $G$.

Using an infinitesimal generator $X$ of the Lie group of transformations $G$, the infinitesimal invariance test can be written in the form:

$$
\underset{m}{X} F\left(x, y, y^{\prime}, \ldots, y^{(m)}\right)=0 \text {. }
$$

Theorem 2.2. [11] For a given $r$-dimensional Lie group $G(r<+\infty)$, acting in the space $(x, y), x \in I \subset \mathbb{R}, y: I \rightarrow \mathbb{R}$ there exists a finite basis of functionally independent invariants and exists operator of the invariant differentiation $Q$ such that an arbitrary fixed order invariant of $G$ can be obtained in a finite number of invariant differentiations and functional operations on invariants from the basis.

Lemma 2.3. [11] Let $\mathcal{A}$ be the Lie algebra of the Lie group $G$ from the Tresse theorem, operators $X_{\nu}=\xi_{\nu}(x, y) \partial_{x}+\eta_{\nu}(x, y) \partial_{y}$ for $\nu=1, \ldots, r$ be the generators of $\mathcal{A}$ and $\xi(x, y)=\left[\xi_{1}, \ldots, \xi_{r}\right]^{T}, \eta(x, y)=\left[\eta_{1}, \ldots, \eta_{r}\right]^{T}$.

Then the properties of the functional basis from the Tresse theorem are the following:

1) This finite basis of invariants is included in the general differential invariant of the minimal order $s \geq 1$ such that:

$$
r=\operatorname{rank}\left[\xi(x, y), \eta(x, y), \zeta^{1}\left(x, y, y^{\prime}\right), \ldots, \zeta^{s-1}\left(x, y, \ldots, y^{(s-1)}\right)\right] .
$$


2) Operator of the invariant differentiation is defined by:

$$
Q=\lambda\left(x, y, y^{\prime}, \ldots, y^{(s)}\right) D_{x},
$$

where $\lambda$ satisfies the commutation condition of operator $Q$ and $X_{\nu}$ :

$$
\underset{s \nu}{X} \lambda=\lambda D_{x}\left(\xi_{\nu}\right)
$$

3) The number of elements in a functional basis of the mth order general invariant (in the case of one function $y$ dependent on one variable $x$ ) is given by the formula

$$
R(m)=m+2-r_{m},
$$

where $r_{m}$ is a rank of the matrix of coefficients of the mth extension of operators $X_{\nu}$.

4) If there exists $k \in \mathbb{N}$ such that all basic invariants of the orders $s-k+1$, $s-k+2, \ldots, s$ can be obtained from basic invariants of the orders $0,1, \ldots$, $s-k$ by a finite number of invariant differentiation and functional operations then the basis of invariants from the Tresse theorem is included in the general invariant of the order $s-k$.

5) The basis of differential invariants together with operators of invariant differentiation from the Tresse theorem of a Lie group $G$ uniquely defines $G$.

Definition 2.4. Let $\Omega$ be a given set of differential equations. An equivalence transformation of $\Omega$ is a nonsingular change of independent and dependent variables preserving $\Omega$, i.e. carrying every equation from $\Omega$ to some equation in $\Omega$. Two equations in $\Omega$ are called equivalent, if there exists an equivalence transformation, carrying one equation to another.

Remark 2.5. [15] Equivalence transformations of a set $\Omega$ of differential equations form a group. If the set $\Omega$ is smooth, the connected component of unit element of this group is a Lie group.

\section{Equivalence transformations of the general Riccati equations}

Let us consider the family of general Riccati equations, described by three arbitrary functions $a(x), b(x), c(x)$ in the form:

$$
y^{\prime}=a(x) y^{2}+b(x) y+c(x) .
$$

Hence, following Definition 2.4, we have

Definition 3.1. Equivalence transformation of the family of general Riccati equations (3.1) is a nonsingular change of variables

$$
\widetilde{x}=\alpha(x, y), \widetilde{y}=\beta(x, y),
$$

preserving the set

$$
\Omega_{R}=\left\{y^{\prime}=a(x) y^{2}+b(x) y+c(x): y, a, b, c: \mathbb{R} \rightarrow \mathbb{R}\right\},
$$


i.e. carrying every equation $y^{\prime}=a(x) y^{2}+b(x) y+c(x)$ from $\Omega_{R}$ to equation

$$
\widetilde{y}^{\prime}=\widetilde{a}(\widetilde{x}) \widetilde{y}^{2}+\widetilde{b}(\widetilde{x}) \widetilde{y}+\widetilde{c}(\widetilde{x}),
$$

where functions $\widetilde{a}, \widetilde{b}, \widetilde{c}$ may be different from $a, b, c$.

Now the infinitesimal technique of finding the Lie algebra of equivalence transformations can be used. Treating $a, b, c$ as new dependent variables we are searching for the symmetry operators

$$
\begin{aligned}
X= & \xi(x, y) \partial_{x}+\eta(x, y) \partial_{y}+\mu^{1}(x, y, a, b, c) \partial_{a}+\mu^{2}(x, y, a, b, c) \partial_{b} \\
& +\mu^{3}(x, y, a, b, c) \partial_{c}
\end{aligned}
$$

of the system of equations

$$
\left\{\begin{array}{l}
y^{\prime}=a y^{2}+b y+c \\
\frac{\partial a}{\partial y}=0, \frac{\partial b}{\partial y}=0, \frac{\partial c}{\partial y}=0
\end{array}\right.
$$

The found operators, which depend also on $a, b, c$ generate a Lie algebra, denoted by $\mathcal{A}$. After integration the generators of $\mathcal{A}$ we obtain the Lie group of equivalence transformations of the set $\Omega_{R}$. Let us denote this Lie group by $G_{R}$.

We calculate the first order extension of operator $X$ to the space $\left(x, y, y^{\prime}, a, a_{x}, a_{y}, b, b_{x}, b_{y}, c, c_{x}, c_{y}\right)$ :

$$
\underset{1}{X}=X+\zeta^{1} \partial_{y^{\prime}}+\nu_{1}^{1} \partial_{a_{x}}+\nu_{2}^{1} \partial_{a_{y}}+\nu_{1}^{2} \partial_{b_{x}}+\nu_{2}^{2} \partial_{b_{y}}+\nu_{1}^{3} \partial_{c_{x}}+\nu_{2}^{3} \partial_{c_{y}},
$$

where

$$
\begin{aligned}
\zeta^{1} & =D_{x}(\eta)-y^{\prime} D_{x}(\xi), \\
\nu_{1}^{1} & =\widetilde{D}_{x}\left(\mu^{1}\right)-a_{x} \widetilde{D}_{x}(\xi)-a_{y} \widetilde{D}_{x}(\eta), \\
\nu_{1}^{2} & =\widetilde{D}_{x}\left(\mu^{2}\right)-b_{x} \widetilde{D}_{x}(\xi)-b_{y} \widetilde{D}_{x}(\eta), \\
\nu_{1}^{3} & =\widetilde{D}_{x}\left(\mu^{3}\right)-c_{x} \widetilde{D}_{x}(\xi)-c_{y} \widetilde{D}_{x}(\eta), \\
\nu_{2}^{1} & =\widetilde{D}_{y}\left(\mu^{1}\right)-a_{x} \widetilde{D}_{y}(\xi)-a_{y} \widetilde{D}_{y}(\eta), \\
\nu_{2}^{2} & =\widetilde{D}_{y}\left(\mu^{2}\right)-b_{x} \widetilde{D}_{y}(\xi)-b_{y} \widetilde{D}_{y}(\eta), \\
\nu_{2}^{3} & =\widetilde{D}_{y}\left(\mu^{3}\right)-c_{x} \widetilde{D}_{y}(\xi)-c_{y} \widetilde{D}_{y}(\eta),
\end{aligned}
$$

and total derivatives are in the form

$$
\begin{aligned}
& D_{x}=\frac{\partial}{\partial x}+y^{\prime} \frac{\partial}{\partial y} \\
& \widetilde{D}_{x}=\frac{\partial}{\partial x}+a_{x} \frac{\partial}{\partial a}+b_{x} \frac{\partial}{\partial b}+c_{x} \frac{\partial}{\partial c} \\
& \widetilde{D}_{y}=\frac{\partial}{\partial y}+a_{y} \frac{\partial}{\partial a}+b_{y} \frac{\partial}{\partial b}+c_{y} \frac{\partial}{\partial c} .
\end{aligned}
$$

Taking into account equations (3.5), we have $\widetilde{D}_{y}=\frac{\partial}{\partial y}$. 
Applying $\underset{1}{X}$ on system (3.5), we obtain the determining equations

$$
\left\{\begin{array}{l}
\zeta^{1}-(2 a y+b) \eta-y^{2} \mu^{1}-y \mu^{2}-\left.\mu^{3}\right|_{(3.5)}=0 \\
\nu_{2}^{1}=\nu_{2}^{2}=\left.\nu_{2}^{3}\right|_{(3.5)}=0
\end{array}\right.
$$

and after analysis of the second equation we have

$$
\mu_{y}^{1}=\mu_{y}^{2}=\mu_{y}^{3}=0 \wedge \xi_{y}=0 .
$$

This means that the transformations of $x, a, b, c$ do not depend on $y$. This fact is also obvious, because supposing opposite, one would obtain more general equations than the Riccati type, i.e. with coefficients depending on $(x, y)$.

The first equation has the form:

$$
\begin{aligned}
& \eta_{x}+\left(\eta_{y}-\xi_{x}\right)\left(a y^{2}+b y+c\right)-\xi_{y}\left(a y^{2}+b y+c\right)^{2}-(2 a y+b) \eta-y^{2} \mu^{1} \\
& \quad-y \mu^{2}-\left.\mu^{3}\right|_{(3.5)}=0 .
\end{aligned}
$$

Here we cannot separate variables, because we have $\eta(x, y), \mu^{i}(x, a, b, c)$. Therefore we differentiate (3.8) with respect to $a, b, c$ and separate variables in the result. After integration of this separated system, by a proper choice of the basis, we obtain the following theorem.

Theorem 3.2. The Lie algebra $\mathcal{A}$ is generated by the operators:

$$
\begin{aligned}
X= & A(x) \partial_{x}+\left(B(x) y^{2}-C(x) y+D(x)\right) \partial_{y} \\
& +\left(\left(C(x)-A^{\prime}(x)\right) a+B(x) b+B^{\prime}(x)\right) \partial_{a}+ \\
& +\left(2 B(x) c-2 D(x) a-A^{\prime}(x) b-C^{\prime}(x)\right) \partial_{b} \\
& +\left(D^{\prime}(x)-D(x) b-\left(A^{\prime}(x)+C(x)\right) c\right) \partial_{c},
\end{aligned}
$$

where $A(x), B(x), C(x), D(x)$ are arbitrary smooth functions.

In all formulas functions $A, B, C, D$ depend on $x$ and in operators we omit it for simplicity. Note that every function $A, B, C, D$ generates some type of equivalence transformations of the family of Riccati equations. Indeed, integrating Lie equations for each operator we obtain the group transformations:

1) $A(x)$ :

$$
X_{A}=A \partial_{x}-A^{\prime} a \partial_{a}-A^{\prime} b \partial_{b}-A^{\prime} c \partial_{c}
$$

Equivalence group transformations are in the form:

$$
\left\{\begin{array}{l}
\widetilde{x}=\widetilde{A}^{-1}(\varepsilon+\widetilde{A}(x))=\alpha(x) \\
\widetilde{y}=y
\end{array}\right.
$$

where $\widetilde{A}(s)=\int \frac{d s}{A(s)}$. This is an arbitrary smooth change of independent variable $x$ with invariant $y$.

2) $B(x)$ :

$$
X_{B}=B y^{2} \partial_{y}+\left(B b+B^{\prime}\right) \partial_{a}+2 B c \partial_{b}
$$


Equivalence group transformations are in the form:

$$
\left\{\begin{array}{l}
\widetilde{x}=x \\
\widetilde{y}=\frac{y}{1-\varepsilon B(x) y} .
\end{array}\right.
$$

Hence such homography of $y$ also preserves the Riccati equation.

3) $C(x)$ :

$$
X_{C}=-C y \partial_{y}+C a \partial_{a}-C^{\prime} \partial_{b}-C c \partial_{c}
$$

Equivalence group transformations are in the form:

$$
\left\{\begin{array}{l}
\widetilde{x}=x \\
\widetilde{y}=y \cdot e^{-\varepsilon \cdot C(x)} .
\end{array}\right.
$$

4) $D(x)$ :

$$
X_{D}=D \partial_{y}-2 D a \partial_{b}+\left(D^{\prime}-D b\right) \partial_{c}
$$

Equivalence group transformations are in the form:

$$
\left\{\begin{array}{l}
\widetilde{x}=x \\
\widetilde{y}=y+\varepsilon \cdot D(x) .
\end{array}\right.
$$

The composition of transformations generated by $X_{A}, X_{B}, X_{C}, X_{D}$ gives the entire Lie group $G_{R}$, because one has

$$
X=X_{A}+X_{B}+X_{C}+X_{D} .
$$

Lemma 3.3. [16] The group of transformations of variable $y$, with invariant $x$ :

$$
y \rightarrow \frac{\alpha(x) y(x)+\beta(x)}{\gamma(x) y(x)+\delta(x)}
$$

with condition $\alpha \delta-\beta \gamma \neq 0$, preserves the Riccati equation.

Theorem 3.4. The Lie algebra $\mathcal{A}$ is a semi-direct sum

$$
\mathcal{A}=\mathcal{A}_{A} \in \mathcal{A}_{B, C, D}=\mathcal{X}(\mathbb{R}) \ltimes C^{\infty}(\mathbb{R}, \operatorname{psl}(2, \mathbb{R})),
$$

where $\mathcal{A}_{B, C, D}$ denotes the Lie algebra generated by operators $X_{B}, X_{C}, X_{D}$.

Hence we have description

$$
G_{R}=\operatorname{Aut}(\mathbb{R}) \ltimes C^{\infty}(\mathbb{R}, P S L(2, \mathbb{R})) .
$$

Moreover, using transformations with one arbitrary function, the orbits of Riccati equation in the form $y^{\prime}=a y^{2}+b y+c$ can be constructed

$$
X_{A}: \widetilde{y}^{\prime}=\frac{a\left(\alpha^{-1}(\widetilde{x})\right)}{\alpha^{\prime}\left(\alpha^{-1}(\widetilde{x})\right)} \cdot \widetilde{y}^{2}+\frac{b\left(\alpha^{-1}(\widetilde{x})\right)}{\alpha^{\prime}\left(\alpha^{-1}(\widetilde{x})\right)} \cdot \widetilde{y}+\frac{c\left(\alpha^{-1}(\widetilde{x})\right)}{\alpha^{\prime}\left(\alpha^{-1}(\widetilde{x})\right)}
$$

where, accordingly $(3.10), \widetilde{x}=\alpha(x)$,

$$
\begin{aligned}
& X_{B}: \widetilde{y}^{\prime}=\left[a+\varepsilon B^{\prime}+\varepsilon B b+\varepsilon^{2} B^{2} c\right] \cdot \widetilde{y}^{2}+(b+2 \varepsilon B c) \widetilde{y}+c, \\
& X_{C}: \widetilde{y}^{\prime}=a e^{\varepsilon C} \cdot \widetilde{y}^{2}+\left(b-\varepsilon C^{\prime}\right) \widetilde{y}+c e^{-\varepsilon C} \\
& X_{D}: \widetilde{y}^{\prime}=a \widetilde{y}^{2}+(b-2 a \varepsilon D) \widetilde{y}+\varepsilon D^{\prime}+a \varepsilon^{2} D^{2}-b \varepsilon D+c .
\end{aligned}
$$


Remark 3.5. Note that not all equivalence transformations can be expressed in explicit form. In the system described above some of transformations may be given by nonintegrable (in quadratures) equations. Therefore in studies of integrability of Riccati equations we use only equivalence transformations given in explicit form.

\section{Invariants of equivalence transformations of the family of Riccati equations}

In this section we investigate invariants of the Lie group of equivalence transformations of the family of Riccati equations. By studying the properties of equation (2.1) for operator (3.9) one can prove that invariants for the entire group $G_{R}$ do not exist. Therefore we find invariants of some important subgroups of this group and by using the Tresse theorem we construct a basis of invariants for these subgroups of transformations. The basis of invariants is a fundamental tool by formulation criteria of equivalence and integrability of the Riccati equations. From all types of found transformations we consider only thirteen subgroups of equivalence transformations, generated by operators:

$$
\begin{gathered}
X_{A}, X_{B}, X_{C}, X_{D},\left(X_{A}, X_{B}\right),\left(X_{A}, X_{C}\right),\left(X_{A}, X_{D}\right),\left(X_{B}, X_{C}\right),\left(X_{C}, X_{D}\right), \\
\left(X_{A}, X_{B}, X_{C}\right),\left(X_{A}, X_{B}, X_{D}\right),\left(X_{A}, X_{C}, X_{D}\right),\left(X_{A}, X_{B}, X_{C}, X_{D}\right) .
\end{gathered}
$$

The choice of such subgroups is motivated by convenience of using criteria depending on differential invariants.

Let us point out that the sets of operators $\left(X_{B}, X_{D}\right)$ and $\left(X_{A}, X_{B}, X_{D}\right)$ are not closed with respect to the Lie bracket and therefore are not considered in this procedure. We construct a basis of invariants and invariant differentiation operator for these subgroup of transformations in the space $\left(x, y, a, b, c, a^{\prime}, b^{\prime}, c^{\prime}, \ldots\right)$, treating all variables as independent. It is easily seen that invariants of some group of transformations are preserved by its subgroups.

According to Definition 2.1 and formula (2.1), we find functions $F$ satisfying the condition:

$$
\underset{m}{X} F\left(x, y, a, b, c, a^{\prime}, b^{\prime}, c^{\prime}, \ldots, a^{(m)}, b^{(m)}, c^{(m)}\right)=0 .
$$

Note that the Lie algebra generated by the operator (3.9) is infinite dimensional. However, after ideas of [11], $\S 24.8$ we can separate variables in above equation over $A, B, C, D$ and their derivatives, which leads the problem to the finite number of dimensions. Further we apply the Tresse theorem directly and construct a basis of invariants and invariant differentiation. Final results are presented in Table 1.

Unfortunately, nontrivial (non-constant) invariants of subgroups of $G_{R}$ generated by operators $\left(X_{A}, X_{B}, X_{C}\right),\left(X_{A}, X_{C}, X_{D}\right),\left(X_{B}, X_{C}, X_{D}\right)$ and $\left(X_{A}, X_{B}, X_{C}, X_{D}\right)$ do not exist. Therefore using invariants, we cannot formulate the necessary and sufficient condition of equivalence of Riccati equations, but only sufficient one. 


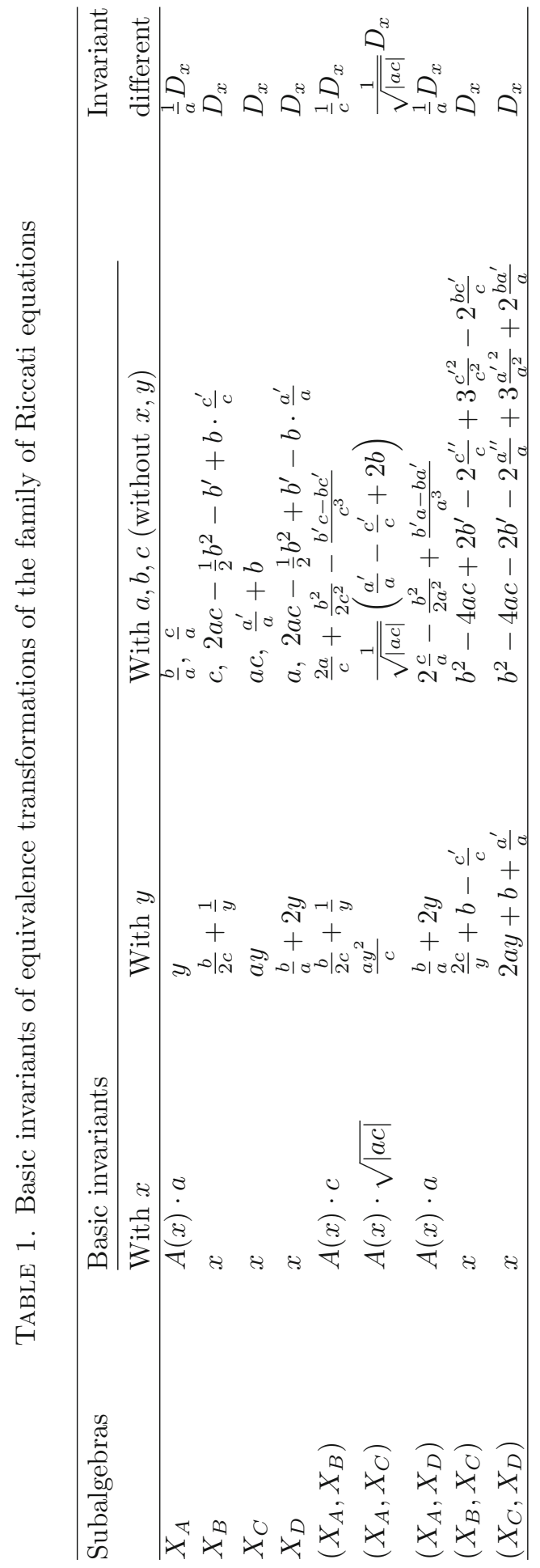


Moreover one can see that transformations generated by $X_{B}$ and $X_{D}$ are conjugated by change of dependent variable $\widetilde{y}=-\frac{1}{y}$. This gives exchange of coefficients $a, c$ and change the sign at $b$. Similarly the transformations generated by $\left(X_{B}, X_{C}\right)$ and $\left(X_{C}, X_{D}\right) ;\left(X_{A}, X_{B}, X_{C}\right)$ and $\left(X_{A}, X_{C}, X_{D}\right)$ are conjugated.

Note that invariants of the equivalence group (or its subgroups), depending on $a, b, c$ can change when $x$ changes, because $a, b, c$ are functions of $x$. This phenomenon has been observed also in [15] for so called Laplace invariants

$$
k=a_{x}+a b-c, \quad h=b_{y}+a b-c
$$

for the class of partial differential equations:

$$
u_{x y}+a(x, y) u_{x}+b(x, y) u_{y}+c(x, y) u=0 .
$$

Such changing "invariants" are called semi-invariants in [15]. What is then really invariant in this case? We introduce invariant combinations of invariants $\omega_{i}$, it means functions $F$ satisfying the condition

$$
F\left(\omega_{1}, \omega_{2}, \ldots, \omega_{k}\right)=\text { const. }
$$

After action of equivalence transformations expressions $\omega_{i}$ can be changed, but their invariant combination $F\left(\omega_{1}, \omega_{2}, \ldots, \omega_{k}\right)$ remain constant, i.e.

$$
F\left(\widetilde{\omega}_{1}, \widetilde{\omega}_{2}, \ldots, \widetilde{\omega}_{k}\right)=F\left(\omega_{1}, \omega_{2}, \ldots, \omega_{k}\right)=\text { const } .
$$

Note that if variable $x$ does not change (for $\left.X_{B}, X_{C}, X_{D}\right)$, invariants $\omega_{i}(x, a, b$, $\left.c, a^{\prime}, b^{\prime}, c^{\prime}, \ldots\right)$ are really invariant.

Lemma 4.1. If invariants $\omega_{i}$ of a given group of transformations depend on $n$ variables then in order to construct invariant combination $F$ satisfying (4.1) it is sufficient to have $n+1$ invariants.

Proof. Suppose we have $n+1$ invariants $\omega_{i}, i=1,2, \ldots, n+1$, depending of $n$ variables $\alpha_{1}, \alpha_{2}, \ldots, \alpha_{n}$, i.e. $\omega_{i}=\varphi_{i}\left(\alpha_{1}, \ldots, \alpha_{n}\right)$. Using the implicit function theorem we can locally determine $\alpha_{1}, \alpha_{2}, \ldots, \alpha_{n}$ from the system

$$
\omega_{i}=\varphi_{i}\left(\alpha_{1}, \ldots, \alpha_{n}\right), \quad i=1,2, \ldots, n
$$

and obtain

$$
\alpha_{j}=\psi_{j}\left(\omega_{1}, \ldots, \omega_{n}\right), \quad j=1,2, \ldots, n .
$$

Then we substitute $\alpha_{j}$ to the equality

$$
\omega_{n+1}=\varphi_{n+1}\left(\alpha_{1}, \ldots, \alpha_{n}\right)
$$

and construct invariant combination:

$$
F\left(\omega_{1}, \ldots, \omega_{n+1}\right)=\omega_{n+1}-\varphi_{n+1}\left(\psi_{1}\left(\omega_{1}, \ldots, \omega_{n}\right), \ldots, \psi_{n}\left(\omega_{1}, \ldots, \omega_{n}\right)\right)=0 .
$$


Lemma 4.2. Every Riccati equation has its own invariant combinations with respect to a given subgroup of equivalence transformations. In general these combinations are different for different equations, but equations equivalent under a given subgroup have the same invariant combinations with respect to this subgroup.

Proof. For a given Riccati equation we have a fixed form of coefficients $a(x)$, $b(x), c(x)$ and invariants $\omega_{i}=\varphi_{i}\left(x, a, b, c, a^{\prime}, b^{\prime}, c^{\prime}, \ldots\right)$. Then it is obvious that the form of the functions $\psi_{j}$ from (4.3) and $F$ from (4.5) may be different for different equations and that a given equation has its own form of $F$. The number of invariant combinations depend on the number of invariants. Using every set of $n+1$ invariants depending on $n$ variables we can construct invariant combination.

Examples of invariant combinations and their applications for miscellaneous Riccati equations are presented in the next section.

\section{Criteria of equivalence of the Riccati equations}

The aim of this section is to formulate criteria of equivalence of two Riccati equations:

$$
y^{\prime}(x)=a_{1}(x) y^{2}(x)+b_{1}(x) y(x)+c_{1}(x)
$$

and

$$
z^{\prime}(t)=a_{2}(t) z^{2}(t)+b_{2}(t) z(t)+c_{2}(t) .
$$

These criteria depend of course on coefficients $a_{1}, b_{1}, c_{1}, a_{2}, b_{2}, c_{2}$ and on their derivatives. Moreover, it is easily seen that equivalence of equations is an equivalence relation, then for a given equation we can describe the class of equations, with which it is equivalent. Because of difficulties pointed out in Remark 3.5 we restrict our considerations to transformations given explicitly.

Theorem 5.1. Equations (5.1) and (5.2) are equivalent with respect to a given subgroup of equivalence transformations if and only if they possess the same all invariants (or invariant combinations) constructed from the basis of invariants for this subgroup of transformations.

Proof. Necessity of this statement is obvious. Sufficiency follows from Lemma 2.3, 5).

For transformations with one arbitrary function is construction of the basis quite a simple problem. Then it is easy to build invariant combinations and investigate the equivalence of Riccati equations. But for transformations with two or more arbitrary functions it is quite complicated problem, because invariants of each subgroup change. In that case, in order to formulate criteria of equivalence, one must compare invariants and invariant combinations of all given transformations together. If invariant combinations of all transformations together are different for two studied equations then they are inequivalent with respect to that subgroup of transformations. 
Example. We investigate the equivalence of the following equations:

1) $y^{\prime}=y^{2}-2 x^{2} y+x^{4}+2 x+4, \quad z^{\prime}=3 t^{2} z^{2}-6 t^{8} z+3 t^{14}+6 t^{5}+12 t^{2}$

2) $y^{\prime}=y^{2}-x y-x, \quad z^{\prime}=z^{2}+z \cdot \frac{3 t-t^{2}}{t-1}+\frac{4 t^{2}-2 t^{3}-t+1}{(t-1)^{2}}$

3) $y^{\prime}=y^{2}+2 x y-3 x^{2}, \quad z^{\prime}=2 t z^{2}+4 t^{3} z\left(t^{2}+1\right)+2 t^{9}+4 t^{7}-6 t^{5}-4 t^{3}$

4) $y^{\prime}=-y^{2}+x^{2}+1, \quad z^{\prime}=-\frac{z^{2}}{t \sqrt{\ln t}}+\frac{z}{2 t \ln t}+\frac{\sqrt{\ln t}}{t}\left(1+\ln ^{2} t\right)$

1) We test invariants of successive subgroups of equivalence transformations, starting from transformations with one arbitrary function. Because $a, c, a c$ change, then it is not transformation, generated by $X_{B}, X_{C}, X_{D}$. Further we study invariants of $X_{A}$ and their invariant combinations for the first equation.

$$
\omega_{11}=\frac{b_{1}}{a_{1}}=-2 x^{2}, \quad \omega_{12}=\frac{c_{1}}{a_{1}}=x^{4}+2 x+4 .
$$

They depend on $x$, hence we calculate invariant combination. We have $x=$ $\sqrt{\left|\frac{\omega_{11} \mid}{-2}\right|}$ and:

$$
\omega_{12}=\frac{\omega_{11}^{2}}{4}+2 \sqrt{\left|\frac{\omega_{11}}{-2}\right|}+4
$$

The invariant combination is:

$$
\Phi\left(\omega_{11}, \omega_{12}\right)=\omega_{12}-\frac{\omega_{11}^{2}}{4}-2 \sqrt{\left|\frac{\omega_{11} \mid}{-2}\right|}=4 .
$$

Now the second equation yields:

$$
\omega_{21}=\frac{b_{2}}{a_{2}}=-2 t^{6}, \quad \omega_{22}=\frac{c_{2}}{a_{2}}=t^{12}+2 t^{3}+4 .
$$

We calculate

$$
\Phi\left(\omega_{21}, \omega_{22}\right)=\omega_{22}-\frac{\omega_{21}^{2}}{4}-2 \sqrt{\left|\frac{\omega_{21} \mid}{-2}\right|}=t^{12}+2 t^{3}+4-t^{12}-2 \sqrt{t^{6}}=4 .
$$

We obtain equality on the basic invariant combination, then using Lemma 2.3 we state that these equations are equivalent. Moreover we can find the change of variables that carries one equation to another using invariant depending on $x$.

$$
x=\sqrt{\left|\frac{\omega_{11}}{-2}\right|}=\sqrt{\left|\frac{\omega_{21}}{-2}\right|}=t^{3} .
$$

One can easily check that this change of $x$ indeed transforms one equation to another.

2) Analogously, we calculate invariants of those transformations and note that coefficient $a$ is the same for these equations and $c, a c$ are different. 
Therefore we check the second basic invariant for $X_{D}$ :

$$
\begin{aligned}
& \omega_{12}=2 a_{1} c_{1}-\frac{1}{2} b_{1}^{2}+b_{1}^{\prime}-b_{1} \cdot \frac{a_{1}^{\prime}}{a_{1}}=-\frac{1}{2}\left(x^{2}+4 x+2\right), \\
& \omega_{22}=2 a_{2} c_{2}-\frac{1}{2} b_{2}^{2}+b_{2}^{\prime}-b_{2} \cdot \frac{a_{2}^{\prime}}{a_{2}}=-\frac{1}{2}\left(t^{2}+4 t+2\right) .
\end{aligned}
$$

The basic invariants are equal for these two equations, hence they are equivalent. The equivalence transformations, generated by $X_{D}$ we find using invariant with $y$ i.e. $2 y+\frac{b}{a}$ :

$$
\begin{aligned}
2 y+\frac{b_{1}}{a_{1}} & =2 z+\frac{b_{2}}{a_{2}}, \\
2 y-x & =2 z+\frac{3 t-t^{2}}{t-1} .
\end{aligned}
$$

Here $x=t$, hence

$$
y=z+\frac{t}{t-1}
$$

Also in this case one can easily check that this transformation carries the first equation into the second.

Moreover one can build an invariant combination using the invariant differentiation. It is the following:

$$
\Phi\left(\omega_{11}, \omega_{12}, \omega_{12}^{\prime}\right)=\omega_{12}+\frac{\omega_{12}^{\prime 2}}{2}=\omega_{22}+\frac{\omega_{22}^{\prime 2}}{2}=1 .
$$

3) In this case we observe, that expressions $a, c, a c$ are not invariant. Also invariant combination for $X_{A}$ is not preserved. It means, that these equations may be equivalent with respect to more than one class of transformations. After calculations of all cases we become convinced that invariant combination of $\left(X_{A}, X_{D}\right)$ is preserved. Indeed:

$\omega_{11}=2 \frac{c_{1}}{a_{1}}-\frac{b_{1}^{2}}{2 a_{1}^{2}}+\frac{b_{1}^{\prime} a_{1}-b_{1} a_{1}^{\prime}}{a_{1}^{3}}=2-8 x^{2}, \quad \omega_{12}=Q\left(\omega_{11}\right)=\frac{1}{a_{1}} \omega_{11}^{\prime}=-16 x$

and we obtain

$$
\Phi\left(\omega_{11}, \omega_{12}\right)=\omega_{11}+\frac{\omega_{12}^{2}}{32}=2 .
$$

For the second equation we have:

$$
\begin{aligned}
\omega_{21} & =2 \frac{c_{2}}{a_{2}}-\frac{b_{2}^{2}}{2 a_{2}^{2}}+\frac{b_{2}^{\prime} a_{2}-b_{2} a_{2}^{\prime}}{a_{2}^{3}}=2-8 t^{4}, \\
\omega_{22} & =Q\left(\omega_{21}\right)=\frac{1}{a_{2}} \omega_{21}^{\prime}=-16 t^{2}, \\
\Phi\left(\omega_{21}, \omega_{22}\right) & =\omega_{22}+\frac{\omega_{21}^{2}}{32}=2 .
\end{aligned}
$$


Now we find the equivalence transformations, using invariants with $x, y$ :

$$
\begin{aligned}
& \omega_{11}=2-8 x^{2} \Longrightarrow \quad x=\sqrt{\frac{2-\omega_{11}}{8}}=\sqrt{\frac{2-\omega_{21}}{8}}=t^{2}, \\
& 2 y+\frac{b_{1}}{a_{1}}=2 z+\frac{b_{2}}{a_{2}} \quad \Longrightarrow \quad 2 y+2 x=2 z+\frac{4 t^{3}\left(1+t^{2}\right)}{2 t} .
\end{aligned}
$$

After substitution $x=t^{2}$ we have:

$$
y=z+t^{4} .
$$

The change of variables: $\left\{\begin{array}{l}x=t^{2} \\ y=z+t^{4}\end{array}\right.$ is the sought equivalence transformation.

4) As above, we check all invariants and invariant combinations and obtain that for $\left(X_{A}, X_{C}\right)$ they are the same. Indeed:

$$
\begin{aligned}
& \omega_{11}=\frac{1}{\sqrt{\left|a_{1} c_{1}\right|}}\left(\frac{a_{1}^{\prime}}{a_{1}}-\frac{c_{1}^{\prime}}{c_{1}}+2 b_{1}\right)=\frac{-2 x}{\left(x^{2}+1\right)^{3 / 2}}, \\
& \omega_{12}=Q\left(\omega_{11}\right)=\frac{1}{\sqrt{\left|a_{1} c_{1}\right|}} D_{x}\left(\omega_{11}\right)=2 \frac{2 x^{2}-1}{\left(x^{2}+1\right)^{3}}, \\
& \Phi\left(\omega_{11}, \omega_{12}\right)=\frac{2 \omega_{11}^{2}}{\omega_{11}^{2}-\omega_{12}}-4 \sqrt[3]{\frac{2}{\omega_{11}^{2}-\omega_{12}}}=-4, \\
& \omega_{21}=\frac{1}{\sqrt{\left|a_{2} c_{2}\right|}}\left(\frac{a_{2}^{\prime}}{a_{2}}-\frac{c_{2}^{\prime}}{c_{2}}+2 b_{2}\right)=\frac{-2 \ln t}{\left(1+\ln ^{2} t\right)^{3 / 2}}, \\
& \omega_{22}=Q\left(\omega_{21}\right)=\frac{1}{\sqrt{\left|a_{2} c_{2}\right|}} D_{x}\left(\omega_{21}\right)=\frac{4 \ln ^{2} t-2}{\left(1+\ln ^{2} t\right)^{3}}, \\
& \Phi\left(\omega_{21}, \omega_{22}\right)=\frac{2 \omega_{21}^{2}}{\omega_{21}^{2}-\omega_{22}}-4 \sqrt[3]{\frac{2}{\omega_{21}^{2}-\omega_{22}}}=-4 .
\end{aligned}
$$

Further we find the equivalence transformations, using invariants with $x, y$ :

$$
\begin{aligned}
x^{2} & =\sqrt[3]{\frac{2}{\omega_{11}^{2}-\omega_{12}}}-1=\sqrt[3]{\frac{2}{\omega_{21}^{2}-\omega_{22}}}=\ln ^{2} t, \\
\frac{a_{1} y^{2}}{c_{1}} & =\frac{a_{2} z^{2}}{c_{2}} \Longrightarrow y=\frac{z}{\sqrt{\ln t}} .
\end{aligned}
$$

The change of variables: $\left\{\begin{array}{l}x=\ln t \\ y=\frac{z}{\sqrt{\ln t}}\end{array}\right.$ is the sought equivalence transformation.

\section{Criteria of integrability of the general Riccati equation}

Criteria of integrability in quadratures are based on criteria of equivalence of a studied equation with some integrable in quadratures equation considering the following lemma: 
Lemma 6.1. If an equation is equivalent with some integrable equation and corresponding equivalence transformations are given in explicit form, then it is also integrable, and if it is equivalent with some nonintegrable equation by such transformations, it is also nonintegrable (in quadratures).

Proof. Note that orbits of equivalence group are disjoint and the action of equivalence group for an equation by explicit transformations preserves the property to be integrable in quadratures.

A well-known method of integration of Riccati equation requires to find a particular solution $y_{0}$ and substitute $y(x)=u(x)+y_{0}$ that reduces a given equation to some Bernoulli equation on $u(x)$, i.e. Riccati equation with $c=0$. Further, by inversion of $u(x)$ one can reduce it to some linear equation (Riccati with $a=0$ ) and integrate, obtaining solution in quadratures.

Hence we can say that this method is based on equivalence of a studied equation with the Bernoulli equation. It is easily seen that any Riccati equation with real coefficients possessing real solutions can be reduced to the Bernoulli equation with real coefficients. Hence we state that the integrability criteria of the Riccati equation are equivalent with criteria of its equivalence with Bernoulli equation. Moreover note that an orbit of the action of equivalence group on Bernoulli equations for equivalence transformations in explicit form gives the class of integrable Riccati equations.

If we put $c=0$ in formulas (3.15), we obtain orbits of Bernoulli equation with respect to transformations with one arbitrary function. Further one can compose transformations with different arbitrary functions and obtain more general form of integrable equations. In practice finding of equivalence transformations carrying one equation to another may be very difficult. Then the knowledge about invariants appears necessary to find them.

Besides Bernoulli equations another two cases of Riccati equations are integrable: equation with $a=0$ (linear equation) and equation with constant coefficients (equation with variables separable). However these cases can be reduced to the Bernoulli equation. Any equation with $a=0$ is equivalent with some equation with $c=0$ by inversion of dependent variable. An equation with constant coefficients can also be reduced to the Bernoulli equation, because one can always find its particular real solution, e.g. as a constant or tangent.

Note that among transformations with one arbitrary function only transformations generated by $X_{D}$ can directly reduce a given Riccati equation to Bernoulli equation. Let consider the Bernoulli equation in the form $y^{\prime}=$ $\alpha(x) y^{2}+\beta(x) y$. Then the Riccati equation $z^{\prime}=a(x) z^{2}+b(x) z+c(x)$ is equivalent with respect to $X_{D}$ with above Bernoulli equation if it has the same basic invariants of $X_{D}$, it means:

$$
a=\alpha \quad \wedge \quad 2 a c-\frac{1}{2} b^{2}+b^{\prime}-b \cdot \frac{a^{\prime}}{a}=-\frac{1}{2} \beta^{2}+\beta^{\prime}-\beta \cdot \frac{\alpha^{\prime}}{\alpha}
$$

All Riccati equations with coefficients $(b, c)$ satisfying (6.1) for given $\alpha, \beta$, form the set of integrable equations, provided that corresponding equivalence transformations are given explicitly. In order to make direct reduction one 
must solve the equation on $D$ :

$$
\varepsilon D^{\prime}+a \varepsilon^{2} D^{2}-b \varepsilon D+c=0 .
$$

Example. In this framework it is possible to answer the questions:

1) For what $c(x)$ equation $z^{\prime}=x z^{2}+z+c$ is equivalent with equation $y^{\prime}=x y^{2}+\frac{1}{x} y ?$

2) For what $b(x)$ equation $z^{\prime}=x^{2} z^{2}+b z+\frac{2}{x^{3}}$ is equivalent with equation $y^{\prime}=x^{2} y^{2}-y ?$

1) We compare invariants and obtain

$$
2 x c=\frac{1}{2}+\frac{1}{x}-\frac{5}{2 x^{2}} \quad \Longrightarrow \quad c=\frac{1}{4 x}+\frac{1}{2 x^{2}}-\frac{5}{4 x^{3}} .
$$

2) Analogously we compare invariants and have

$$
b^{\prime}=\frac{1}{2} b^{2}+\frac{2}{x} b-\frac{2}{x}-\frac{1}{2} .
$$

Observing, that $b_{0}(x)=1$ is the particular solution, we can solve it in general

$$
b(x)=1+\frac{x^{2} e^{x}}{k-\frac{1}{2} x^{2} e^{x}+x e^{x}-e^{x}},
$$

where $k$ is an arbitrary constant.

If we consider equivalence transformations generated by $\left(X_{A}, X_{D}\right)$ and their invariants we obtain the condition:

$$
2 \frac{c}{a}-\frac{b^{2}}{2 a^{2}}+\frac{b^{\prime} a-b a^{\prime}}{a^{3}}=-\frac{\beta^{2}}{2 \alpha^{2}}+\frac{\beta^{\prime} \alpha-\beta \alpha^{\prime}}{\alpha^{3}}
$$

Also Riccati equations with coefficients $(a, b, c)$ satisfying (6.3) form the set of integrable equations, provided that corresponding equivalence transformations are explicit. Now one can fix two coefficients and calculate the third one. However in this case variable $x$ changes and it is possible, that equations are equivalent and invariants are different. Therefore in order to investigate the equivalence one must compare invariant combination of invariants.

For transformations generated by $\left(X_{C}, X_{D}\right)$ we have the condition:

$b^{2}-4 a c-2 b^{\prime}-2 \frac{a^{\prime \prime}}{a}+3 \frac{a^{\prime 2}}{a^{2}}+2 \frac{b a^{\prime}}{a}=\beta^{2}-2 \beta^{\prime}-2 \frac{\alpha^{\prime \prime}}{\alpha}+3 \frac{\alpha^{\prime 2}}{\alpha^{2}}+2 \frac{\beta \alpha^{\prime}}{\alpha}$

Also in this case Riccati equations with coefficients $(a, b, c)$ satisfying (6.4) form the set of integrable equations, if equivalence transformations are explicit.

Therefore conditions (6.1)-(6.4) have a nature of integrability criteria of the Riccati equation and they are formulated by means of invariants of equivalence group. 


\section{Concluding remarks}

- By using the group $G_{R}$ and its invariants, the criteria of equivalence and integrability in quadratures of the Riccati equations have been formulated. In this approach one can also generate classes of integrable and nonintegrable Riccati equations.

- We investigated the necessary and sufficient conditions of integrability in quadratures in the real domain. The investigations of the equivalence transformations of the Riccati equations in the complex domain would also be interesting.

- Criteria (6.1)-(6.4) can be further used in investigations of integrability of Riccati equation in many ways, e.g. they can be reformulated according to Lemma 6.1, it means instead of Bernoulli equation one can use some integrable Riccati equation with $c \neq 0$.

- This approach can be used for ascertainment of equivalence and integrability of other important differential equations, ordinary as well as partial ones.

\section{Acknowledgments}

J. H. is grateful for partial support by the Ministry of Education of Czech Republic (project MSM6840770039). T. Cz. is grateful to prof. I. Tsyfra for searching discussions, especially about differential invariants and idea of invariant combinations.

\section{References}

[1] Popovych, R.O., Boyko, V.M.: Differential invariants and application to Riccati-type systems. Proc. Inst. Math. NAS Ukraine 43(1), 184-193 (2002)

[2] Lie, S.: Klassifikation und Integration von gew onlichen Differentialgleichungen zwischen x, y, die eine Gruppe von Transformationen gestaten. IV, Archiv for Matematik og Naturvidenskab., vol. 9, Heft 4, pp. 431-448 (1884) [reprinted in Lie's Ges. Abhandl., vol. 5, paper XVI, pp. 432-446 (1924)]

[3] Ovsiannikov, L.V.: Group properties of the equation of S.A. Chaplygin. J. Appl. Mech. Tech. Phys. 3, 126-145 (in Russian) (1960)

[4] Ovsiannikov, L.V.: Group properties of differential equations. Siberian Branch of USSR Academy of Sciences, Novosibirsk (in Russian) (1962)

[5] Boyko, V.M.: Symmetry, equivalence and integrable classes of Abel equations. 4 July 2005. arXiv:nlin.SI/0404020 v2

[6] Olver, P.J.: Differential invariants and invariant differential equations. Lie Groups Appl. 1, 177-192 (1994) 
[7] Chen, J., Olver, P.J., Pohjanpelto, J.: Algorithms for differential invariants of symmetry groups of differential equations. Found. Comput. Math. (2009, to appear). http://www.math.umn.edu/ olver/xtra.html

[8] Czyżycki, T.: The Tresse theorem and differential invariants for the nonlinear Schrödinger equation. J. Phys. A Math. Theor. 40, 9331-9342 (2007)

[9] Zhdanov, R.Z., Tsyfra, I.M., Popovych, R.O.: A Precise Definition Of Reduction Of Partial Differential Equations. J. Math. Anal. Appl. 238, 101-123 (1999)

[10] Novotný, P., Hrivnák, J.: On $(\alpha, \beta, \gamma)$-derivations of Lie algebras and corresponding invariant functions. J. Geom. Phys. 58(2), 208-217 (2008)

[11] Ovsiannikov, L.V.: Group Analysis of Differential Equations. Academic Press, New York (1982)

[12] Olver, P.J.: Applications of Lie Groups to Differential Equations. Springer, New York (1986)

[13] Olver, P.J.: Equivalence, Invariants and Symmetry. Cambridge University Press, Cambridge (1995)

[14] Ibragimov, N.H.: Group of Transformations in Mathematical Physics. Nauka, Moscow (1983)

[15] Ibragimov, N.H.: Equivalence groups and invariants of linear and non-linear equations Archives of ALGA, vol. 1. Blekinge Institute of Technology, Karlskrona, Sweden (2004)

[16] Matveyev, M.N.: Methods of Integrating Ordinary Differential Equations. Polish Science Publishers, PWN Warsaw (in Polish, translation from Russian) (1980)

T. Czyżycki

Institute of Mathematics,

University of Bialystok,

Białystok, Poland

e-mail: tomczyz@math.uwb.edu.pl

J. Hrivnák

Department of Physics,

Faculty of Nuclear Sciences and Physical Engineering,

Czech Technical University,

Prague, Czech Republic

e-mail: hrivnak@fjfi.cvut.cz

Received: 22 October 2008.

Accepted: 30 October 2009. 